Conflict of interest: ALC has a patent pending for Method for High Percentage Recovery of Rare Cells (European application 18185007.4). MSW receives research support from the DFG (WE 3547/5-1), Novartis, Teva Pharmaceutical Industries, Biogen Idec, Roche, Merck, and the ProFutura Program of the Universitätsmedizin Cöttingen. MSW is serving as an editor for PLoS One. He received travel funding and/ or speaker honoraria from Biogen Idec, Merck Serono, Novartis, Roche/Genentech, Teva Pharmaceutical Industries, Bayer, and Sanofi Genzyme. RGH has received research funding from Roche/ Genentech and Sanofi Cenzyme. BAC has received personal compensation for consulting from EMD Serono and Novartis. SLH currently serves on the scientific advisory board of Symbiotix, Annexon, Bionure, Molecular Stethoscope, and Alector and on the board of trustees of Neurona. SLH also has received travel reimbursement and writing assistance from F. Hoffmann-La Roche Ltd. for CD20-related meetings and presentations. MRW has received research support from Roche/Genentech and has a patent pending for Method for High Percentage Recovery of Rare Cells (European application 18185007.4). HCVB is an employee of F. Hoffmann-La Roche Ltd., Basel, Switzerland (Roche) and reports equity in Roche and income as full-time employee.

Copyright: $\odot 2019$ American Society for Clinical Investigation

Submitted: December 5, 2018 Accepted: February 5, 2019 Published: March 21, 2019.

Reference information: $\mathrm{JCl}$ Insight. 2019;4(6):e126599. https://doi.org/10.1172/jci. insight.126599.

\section{Longitudinally persistent cerebrospinal fluid B cells can resist treatment in multiple sclerosis}

\author{
Ariele L. Greenfield, ${ }^{1}$ Ravi Dandekar, ${ }^{1}$ Akshaya Ramesh, ${ }^{1}$ Erica L. Eggers, ${ }^{1} \mathrm{Hao}$ Wu, ${ }^{1}$ \\ Sarah Laurent, ${ }^{1}$ William Harkin, ${ }^{1}$ Natalie S. Pierson, ${ }^{1}$ Martin S. Weber, ${ }^{2}$ Roland G. Henry, ${ }^{1}$ \\ Antje Bischof, ${ }^{1}$ Bruce A.C. Cree, ${ }^{1}$ Stephen L. Hauser, ${ }^{1}$ Michael R. Wilson, ${ }^{1}$ \\ and $\mathrm{H}$.-Christian von Büdingen ${ }^{1}$ \\ 'UCSF Weill Institute for Neurosciences, Department of Neurology, UCSF, San Francisco, California, USA. ${ }^{2}$ Institute of \\ Neuropathology, Department of Neurology, University Medical Center Göttingen, Germany.
}

B cells are key contributors to chronic autoimmune pathology in multiple sclerosis (MS). Clonally related B cells exist in the cerebrospinal fluid (CSF), meninges, and CNS parenchyma of MS patients. We sought to investigate the presence of clonally related B cells over time by performing Ig heavy chain variable region repertoire sequencing on B cells from longitudinally collected blood and CSF samples of MS patients $(n=10)$. All patients were untreated at the time of the initial sampling; the majority $(n=7)$ were treated with immune-modulating therapies $1.2( \pm 0.3 \mathrm{SD})$ years later during the second sampling. We found clonal persistence of B cells in the CSF of 5 patients; these $B$ cells were frequently Ig class-switched and $\mathrm{CD} 27^{+}$. Specific blood B cell subsets appear to provide input into CNS repertoires over time. We demonstrate complex patterns of clonal B cell persistence in CSF and blood, even in patients on immune-modulating therapy. Our findings support the concept that peripheral B cell activation and CNS-compartmentalized immune mechanisms can in part be therapy resistant.

\section{Introduction}

B lymphocytes (B cells) are key players in the immunopathology of multiple sclerosis (MS) (1-6). B cell-depleting anti-CD20 antibody therapies effectively reduce relapsing MS disease activity and slow the accumulation of disability in relapsing and primary progressive disease (2, 3, 7). Early on in the disease course, B cells enter the CNS, cerebrospinal fluid (CSF), and meningeal compartments (8-10), where they become compartmentalized and likely contribute to ongoing CNS tissue damage and consequential disability progression.

Ig gene transcripts, particularly heavy chain variable regions $(\mathrm{Ig}-\mathrm{VH})$, provide molecular fingerprints that permit temporal and spatial tracking of clonally related B cells (11-13). Single-time point immune repertoire studies in MS patients showed that there is an anatomic continuum of clonally related B cells extending from MS lesions in the brain parenchyma to CSF, meningeal inflammatory infiltrates, cervical lymph nodes, and peripheral blood (PB) (11, 12, 14-16). Individual MS patients have a characteristic CSF Ig electrophoretic pattern (oligoclonal bands [OCBs]) that persists over many years; the stability in the pattern of OCBs within a patient is considered evidence that clonally related B cells are long-lived in the CNS $(17,18)$. However, because multiple B cell clones can give rise to a single OCB $(16)$, higher resolution genomic techniques are required to confirm this hypothesis.

Here, we were interested to determine the persistence of clonally related B cells in the CSF and in $\mathrm{PB}$ over time. Using previously described immune repertoire sequencing technology and bioinformatics tools (8), we analyzed Ig-VH sequences (IgG-VH and IgM-VH) (19) from 10 patients' PB and CSF B cell subsets at 2 time points to track related B cells over time and across compartments. Work by others previously described 2 untreated MS patients with clonally related CSF B cells over time (20). Here, we were interested to understand which specific B cell subsets comprise clonally related CSF B cells that remain longitudinally detectable, their clonal relationship to PB B cells, and whether CSF B cells persist after treatment with MS immune-modulating therapy (IMT). We identified persistent, clonally related B cells in the CSF of 5 of the 10 patients, 4 of whom had initiated IMT between the first and second 
time points. We also identified patterns of clonal B cell input from the periphery to the CSF over time, suggesting that functionally diverse $\mathrm{CD} 27^{+} \mathrm{PB}$ memory B cells are a likely peripheral reservoir of B cells involved in MS disease activity.

\section{Results}

Patient demographics. Ten patients ranging in age from 24 to 52 years old were enrolled (see Table 1 for clinical data and Supplemental Table 1 [supplemental material available online with this article; https:// doi.org/10.1172/jci.insight.126599DS1] for standard laboratory CSF findings). On average, $12.2 \mathrm{ml}$ ( \pm 3.5 $\mathrm{SD})$ of CSF was collected per patient per time point. Eight patients had relapsing-remitting MS (RRMS), and 2 had primary progressive MS (PPMS). All were untreated at time point 1 (T1), and 7 of the 8 RRMS patients were on IMT by time point 2 (T2) (Table 1). Because there were no approved IMTs for PPMS before 2017, neither PPMS patient was on an IMT. IMTs used in this cohort varied from lower efficacy to higher efficacy treatments. Eight of 10 patients ( 6 treated, 2 untreated) had enhancing or new lesions on brain or spinal cord MRI at T2 (Table 1). All patients had OCBs unique to the CSF at both time points (Supplemental Table 2). In 7 of 10 patients, we were able to directly compare the OCB pattern at each time point (Supplemental Figure 1): 5 of 7 patients had stable band patterns, 1 had a decrease in band number, and 1 had an increase in band number (Supplemental Figure 1 and Supplemental Table 2).

Flow cytometry. B cell subsets were identified and sorted by flow cytometry (MoFlo Astrios EQ). Flow cytometric B cell subset distribution (see Methods) was determined at T1 and T2 for $n=8$ patients' CSF and for $n=9$ patients' PB; flow cytometry was not available for time point 1 CSF (T1-CSF) and peripheral blood (T1-PB) of 1 patient and for both CSF time points of another patient. When compared with $\mathrm{PB}$, the $\mathrm{CSF}$ was enriched in $\mathrm{CD}_{19}{ }^{+} \mathrm{CD} 27^{+} \mathrm{IgD} \mathrm{D}^{-}$Ig class-switched memory (SM) B cells (Supplemental Figure 2), consistent with previous reports $(8,21,22)$.

Immune repertoire sequencing. IgG-VH and/or IgM-VH repertoire sequencing cDNA libraries were prepared from 167 samples. Samples consisted of PB or CSF FACS-sorted B cell subsets or, alternatively, bulk CSF or PB mononuclear cells (Supplemental Table 3). Sequencing libraries could not be obtained from 16 samples (Supplemental Table 3). From the remaining 151 samples, we generated 583,932 $( \pm 652,920 \mathrm{SD})$ raw reads per library. We identified $218,401( \pm 308,602 \mathrm{SD})$ Ig-VH sequences per library from the Ig heavy chain variable germline segment (IGHV), Ig heavy chain joining germline segment (IGHJ) and Ig heavy chain complementarity-determining region 3 (H-CDR3) for further analysis (Supplemental Table 3). Ig-VH sequences were clustered using a distance metric approach (see Methods). Samples with more B cells had more Ig-VH clusters (Supplemental Figure 3) $(r=0.88, P<0.0001$ for all samples; $r=0.74, P<0.0001$ for $\mathrm{PB} ; r=0.59, P<0.0001$ for CSF, Spearman's correlation). Five paucicellular B cell subsets yielded more Ig-VH clusters than the number of input cells (Supplemental Table 3). For these samples, we analyzed the same number of Ig-VH clusters as input cells, choosing the Ig-VH clusters with the greatest number of aligned sequencing reads. Mutational analyses within Ig-VH clusters were not performed because these were not needed for the conclusions of this study.

At T1, we identified CSF Ig-VH clusters that were exclusively IgG-VH in all 10 patients $(26.4[ \pm 28.3$ $\mathrm{SD}$ ] Ig-VH clusters/patient); of the 10 patients, 9 patients also had CSF Ig-VH clusters that contained exclusively IgM-VH (44.8 [ $\pm 57.3 \mathrm{SD}] \mathrm{Ig}-\mathrm{VH}$ clusters/patient), and in 5 patients, we found mixed IgM and IgG clusters (5.2 [ $\pm 9.4 \mathrm{SD}]$ Ig-VH clusters/patient) (Supplemental Figure 4). At T2, we found that all 10 patients' CSF contained Ig-VH clusters that were exclusively IgG-VH (42.6 [ $\pm 72.6 \mathrm{SD}] \mathrm{Ig}-\mathrm{VH}$ clusters/patient) or exclusively IgM-VH (31.7 [ $\pm 33.9 \mathrm{SD}] \mathrm{Ig}-\mathrm{VH}$ clusters/patient); in 7 patients, there were 6.7 ( $\pm 11.8 \mathrm{SD}$ ) Ig-VH clusters/patient that were mixed IgM and IgG (Supplemental Figure 4). At T1, SM and naive B cells were common members of CSF Ig-VH repertoire clusters: These subsets were prevalent in repertoires of 3 out of 5 and 2 out of 5 patients with sorted T1-CSF B cells, respectively (59.6 [ $\pm 80.4 \mathrm{SD}]$ Ig-VH clusters/patient, 60.8 [ $\pm 65.9 \mathrm{SD}$ ] Ig-VH clusters/patient, respectively) (Supplemental Figure 5). SM B cells commonly contributed to T2-CSF: 5 of 8 patients with sorted T2-CSF B cells had SM-predominant repertoires (45.4 [ $\pm 65.9 \mathrm{SD}]$ Ig-VH clusters/patient) (Supplemental Figure 5).

Clonally related $B$ cells persist in MS CSF. In 5 of 10 patients, we identified "persistent CSF Ig-VH clusters" in which CSF Ig-VH sequences from both time points were represented (Figures 1-3); we thus demonstrate that B cells found in MS patients' CSF at different time points are clonally related. Aside from Ig-VH sequences that exclusively persisted in CSF (Supplemental Figure 6), we identified 3 possible associations of CSF Ig-VH clusters with PB repertoires: a T1-PB connection, a T2-PB connection, or 
Table 1. Patient demographics

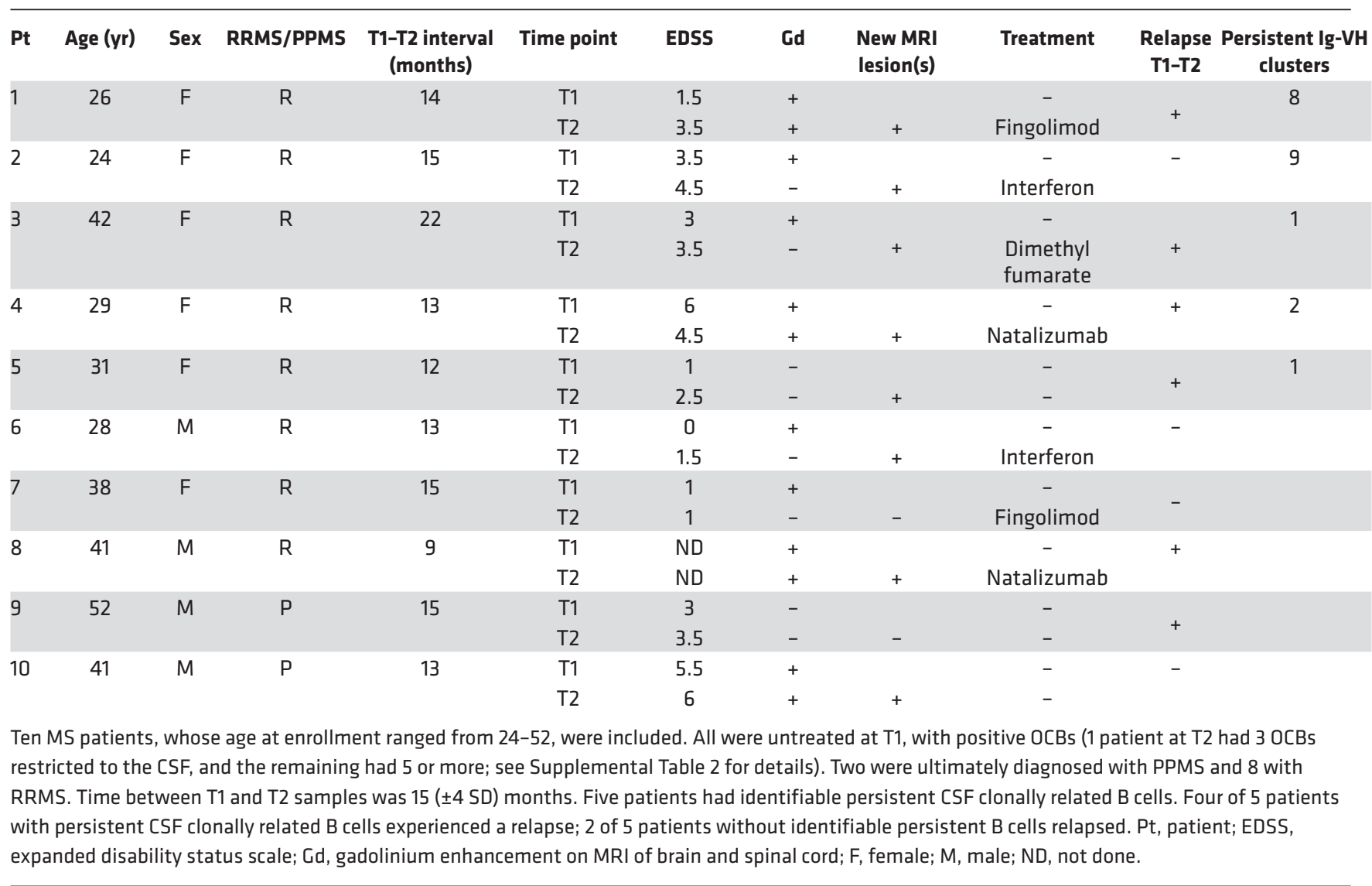

connections with both PB time point samples (Figure 2). We found IgG-expressing B cells, including SM $\mathrm{B}$ cells and plasmablast/plasma cells (PCs), in persistent CSF Ig-VH clusters of all 5 patients with persistent CSF Ig-VH clusters (Figure 3). In contrast, IgM-expressing B cell subsets were found to take part in persistent CSF Ig-VH clusters in only 2 patients (patients 1 and 3) (Figure 3). In particular, we did not find naive CSF B cells in persistent CSF Ig-VH clusters.

There was no relationship between the total CSF white blood cell count (clinical diagnostic that includes all leukocytes, not just B cells) at T1 or T2 and the number of persistent CSF Ig-VH clusters $\left(r^{2}\right.$ $=0.32, P=0.09$, and $r^{2}=0.07, P=0.47$, respectively; not shown). There was no significant correlation between the detection of persistent CSF Ig-VH clusters over time and the following clinical characteristics: age, disease duration, time between T1 and T2, EDSS score, use of IMT, gadolinium enhancement on MRI at either time point, IgG index, relapse between T1 and T2, or number of OCBs (Supplemental Tables 1 and 2). More of the patients without persistent CSF Ig-VH clusters were men (Supplemental Table 1). There was a numerical (yet not statistically significant) imbalance in the number of patients with relapses in that more of the patients with identified persistent CSF Ig-VH clusters experienced a relapse. There was no significant difference between B cell subset distribution in patients with or without persistent CSF Ig-VH clusters (Supplemental Figure 7). We found no correlation between the average reads per cell and our ability to detect persistent CSF Ig-VH clusters ( $r=0.2$, Spearman's correlation; $P=0.17$ ). The percentage of Ig-VH clusters in CSF that were persistent was similar to the percentage of Ig-VH clusters that were persistent in PB (i.e., Ig-VH clusters formed from PB at both time points) (Supplemental Table 4). This suggests that immune processes such as antigen stimulation and B cell regulation, which maintain a proportion of B cell clonal populations in the periphery, may also occur in the CNS.

$P B S M$ and unswitched memory B cells are potential contributors to CSF B cell repertoires. Clonal connections between $\mathrm{PB}$ and CSF were identified in all patients, irrespective of whether persistent CSF Ig-VH clusters or clonal relatedness within the same $\mathrm{PB}$ and CSF subset was present (Figure 3 and 


\section{With CSF persistence}

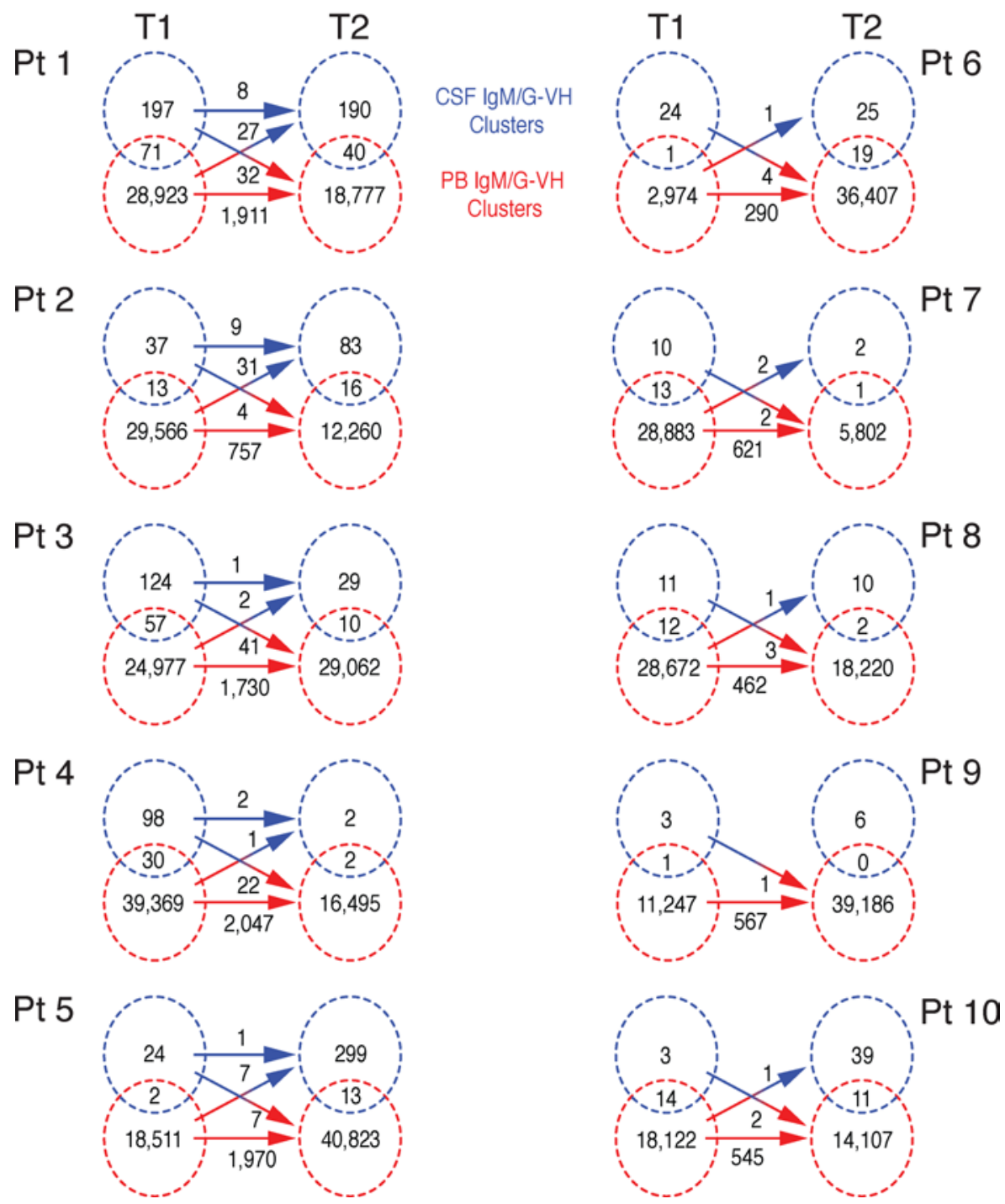

Figure 1. Persistent CSF Ig-VH clusters are present in MS patients. Shown are the numbers of Ig-VH clusters within CSF (blue circles) and PB (red circles) at T1 and T2. Circle overlap values, Ig-VH clusters found in both CSF and PB at T1 or T2; nonoverlap circle values, Ig-VH clusters exclusively found in CSF or PB, not both. Arrows, of total Ig-VH clusters in CSF or PB (nonoverlap portion of circle + circle overlap), the number of lg-VH clusters that are present at both $\mathrm{T} 1$ and $\mathrm{T} 2$. Patients (Pts) 1-5 have persistent CSF Ig-VH clusters; Pts 6-10 did not have identifiable persistent CSF Ig-VH clusters.

Supplemental Figure 8). Therefore, to understand which PB B cell subsets might contribute clonal input to CSF repertoires over time, we examined Ig-VH clusters containing T1-PB and T2-CSF members yet no T1-CSF members. In all 9 patients with sorted T1-PB subsets, T1-PB unswitched memory (USM) (6 patients) and SM (5 patients) B cells were related to T2-CSF (Figure 3 and Supplemental Figure 8). All of these patients except for 1 (patient 5) were treated with IMT by T2. Other T1-PB $\mathrm{B}$ cell subsets sharing repertoires with T2-CSF included naive (4 patients), double negative (DN) (3 patients), and PC (3 patients) (Figure 3 and Supplemental Figure 8). Although overall somatic hypermutation (SHM) rates followed expected patterns for B cell subsets based on maturation stage, certain CSF subsets, such as IgG SM in patient 1, were noted to have a particularly high degree of SHM - suggesting affinity maturation (Supplemental Figure 9). Whether Ig class-switching occurs in USM B cells has been debated (23); here we found numerous Ig-VH clusters containing both USM $\left(\mathrm{CD}^{2} 7^{+} \mathrm{IgD}^{+} \mathrm{IgM}^{+}\right)$and class-switched $\mathrm{IgG}^{+} \mathrm{B}$ cells (i.e., $\mathrm{SM}, \mathrm{PC}$, and $\left.\mathrm{DN}\right)$ in $\mathrm{CSF}$ and $\mathrm{PB}(2.5[ \pm 2.6$ SD] Ig-VH clusters/patient in CSF, 125.6 [ \pm 96.6 SD] Ig-VH clusters/patient in PB) (Supplemental 


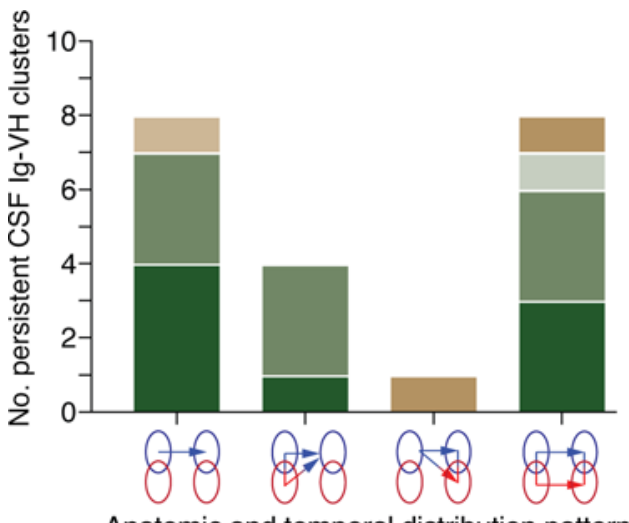

Anatomic and temporal distribution pattern

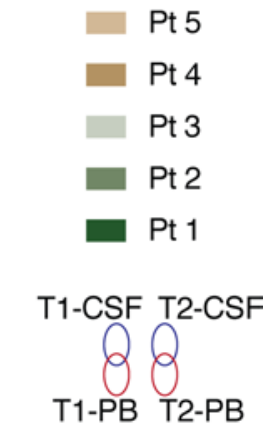

Figure 2. Persistent CSF Ig-VH clusters have multiple patterns of persistence in relation to PB. Persistent CSF Ig-VH clusters can be exclusive to CSF (far left, Pts 1, 2, 5), link to T1-PB (second from left, Pts 1, 2), link to T2-PB (second from right, Pt 4), or link to both PB time points (far right, Pts 1-4). Blue circles, CSF; red circles, PB; circles on left, T1; circles on right, T2; arrows, shared Ig-VH clusters between time points or between CSF and PB.

Figure 10). To explore whether CSF PCs might be derived from long-lived PB PC populations, we searched for, but did not find, persistent PB PCs that were clonally related to CSF PCs. Incidentally, we found a frequent occurrence of persisting IgM-VH clusters with $\mathrm{PB}$ naive $\mathrm{B}$ cell members at $\mathrm{T} 1$ and $\mathrm{T} 2$, suggesting long-term survival and clonal expansion of naive B cells; previously, naive B cells have been characterized as short-lived precursors to memory and plasmablasts/PCs (24).

\section{Discussion}

We identified clonally related B cells in CSF that were detectable at time points between 9 and 22 months apart. Given the histopathological and biochemical evidence for B cell recruitment, survival, and proliferation in the CNS compartment (25-27), the ability to detect clonally related CSF B cells by immune repertoire sequencing over time is likely the result of local persistence (i.e., long-term survival) of B cells in the functionally connected CNS, CSF, and meningeal compartments. This is perhaps consistent with the disease activity observed in some patients examined in this study and is also consistent with prior findings of antigen-experienced B cells and soluble Ig in the CSF that suggests persistence over time. These cells could constitute a tissue-resident immune repertoire capable of being restimulated - either directly or by bystander activation - to rekindle inflammation, mechanisms that may be shared with rheumatoid arthritis and other tissue-specific chronic autoimmune diseases (28). B cells of persistent CSF Ig-VH clusters display phenotypic features indicative of antigen-driven immunity (i.e., CD27-expression and Ig classswitched B cell receptors). Persistent B cells in $\mathrm{PB}$, on the other hand, belong to a less restricted set of B cell subsets and include apparently long-lived naive $\left(\mathrm{IgD}^{+} \mathrm{CD} 27^{-}\right) \mathrm{B}$ cell populations in addition to memory $\mathrm{B}$ cell and PC subsets. Overall, persistent CSF B cells comprise a minority of the overall CSF B cell diversity. Nonetheless, their persistence despite IMT initiation suggests that these B cell populations could be representative of a therapy-resistant, CNS-compartmentalized reservoir of immune cells. OCBs as well are evidence for CNS/CSF compartmentalization, albeit only of PCs as producers of soluble Ig. CNS-compartmentalized B cells are believed to contribute to progressive CNS tissue damage in MS (9, 25, 29); identification of CSF-persistent, functionally diverse B cell subsets (e.g., memory B cells, PCs) suggests that multiple aspects of active adaptive immunity are reflected in the CNS that are capable of supporting the immunopathogenesis of MS in various ways (i.e., cytokine secretion, antigen presentation, and antibody production). Interestingly, even after long-term high-efficacy IMT $(30,31)$, including intrathecal rituximab (32), patients continue to be at risk of progression. Indeed, our findings lend further support to the hypothesis that currently available IMTs may not sufficiently target progression-promoting mechanisms located in the CNS compartment. B cell-depleting therapies do not affect CD20- PCs, and oligoclonal antibodies persist in CSF of rituximab-treated patients $(33,34)$; future studies are needed to determine whether antiCD20 therapies deplete persistent clonal B cells from the CSF, CNS, and meninges and whether doing so reduces disease progression. Our findings may suggest that development of novel therapies that target CNS-compartmentalized immune mechanisms could help close an important therapeutic gap. 
A

Pt 1
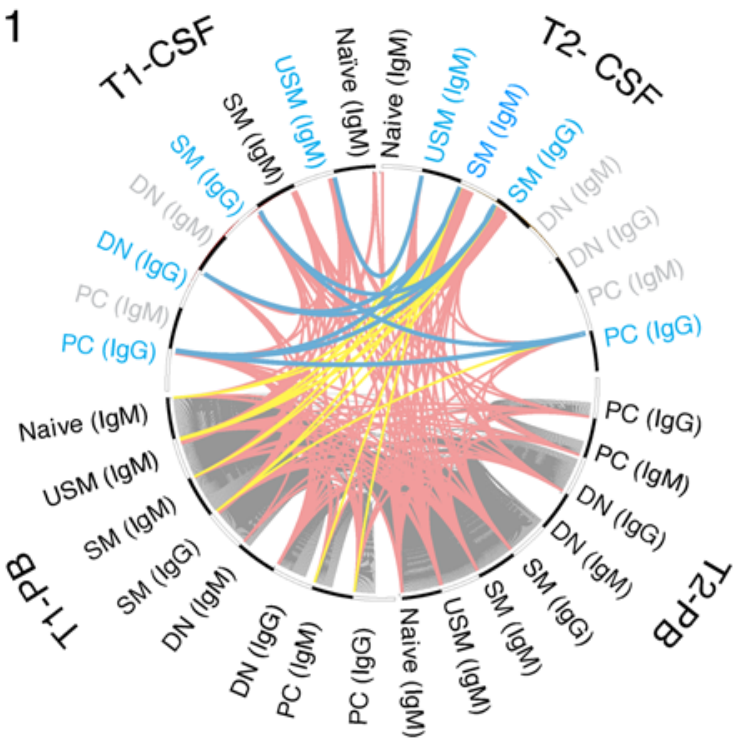

Pt 3

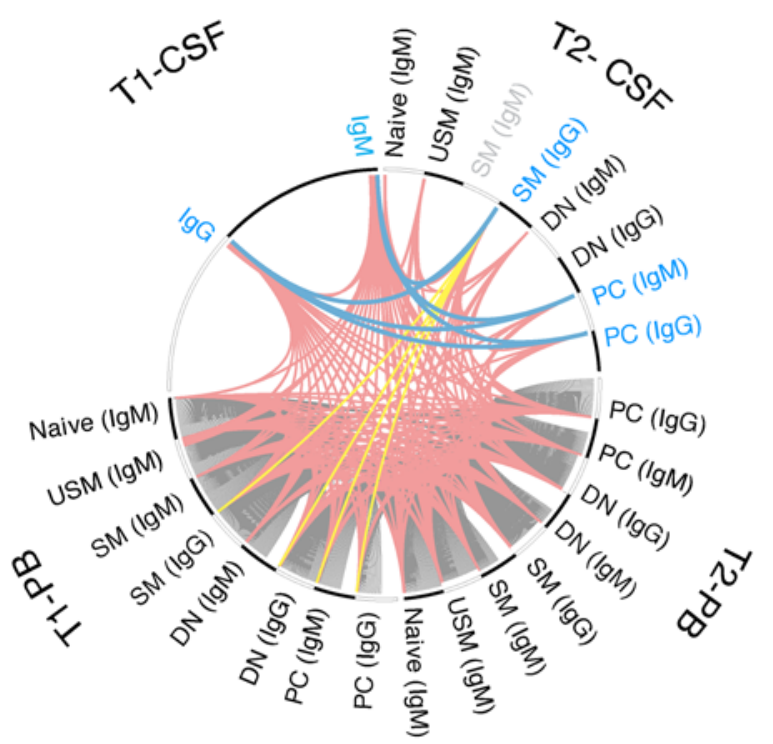

Pt 5

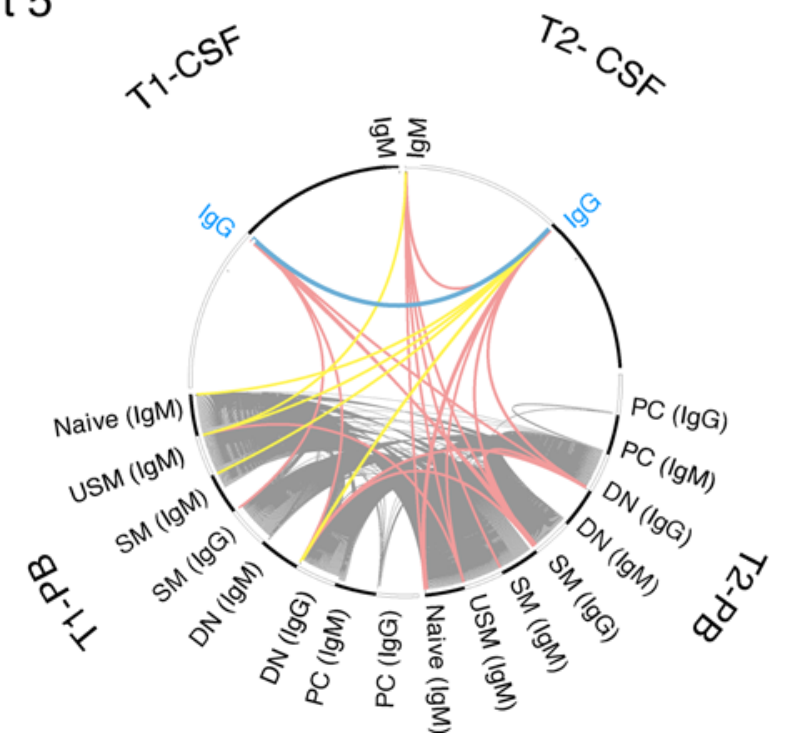

\section{Pt 2}

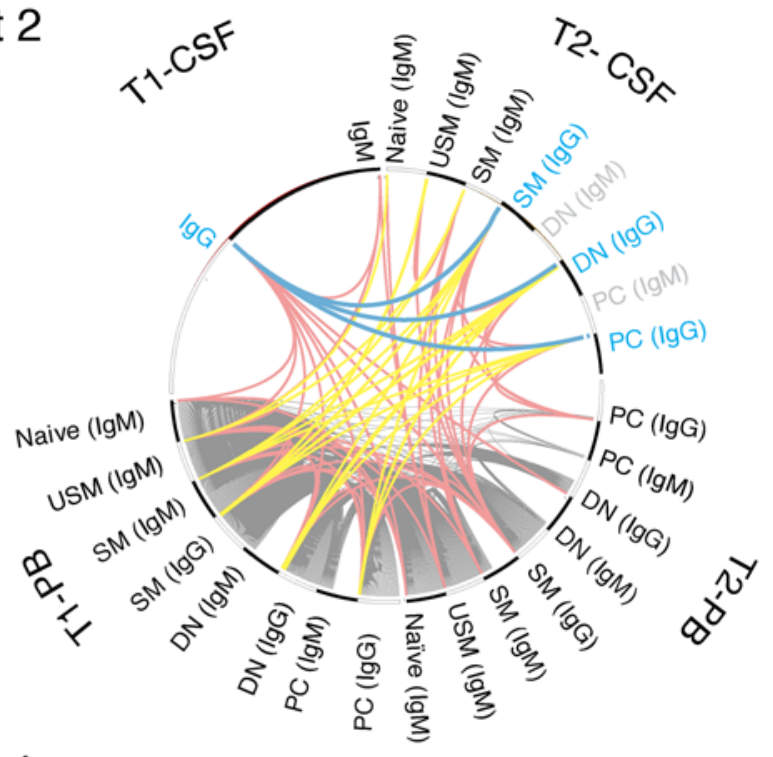

Pt 4

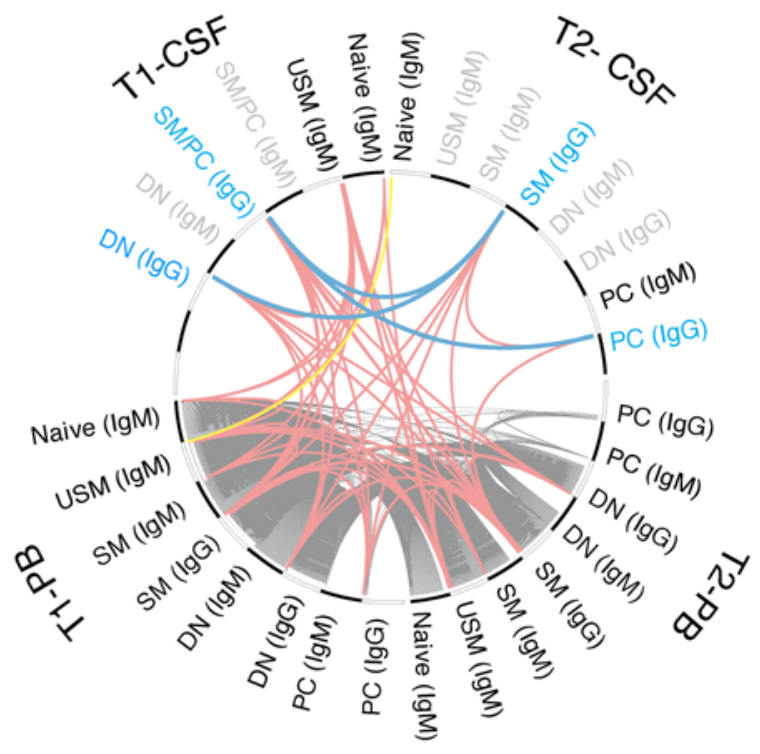

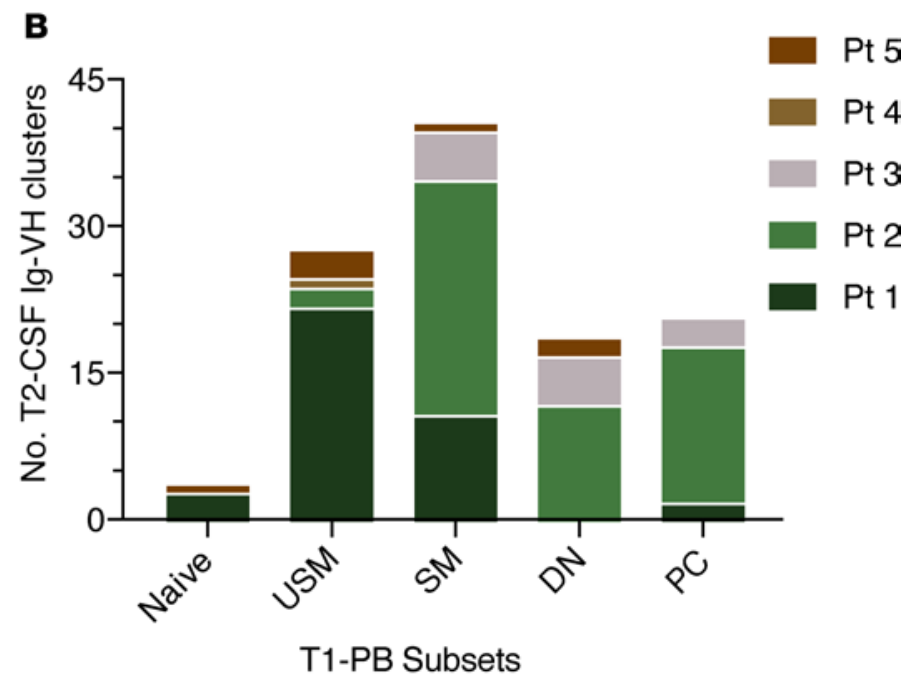


Figure 3. B cells that persist in the CSF are often SM and plasma cells. (A) Clonally related B cell subsets are connected by lines between CSF (top half of circle) and PB (bottom half) at T1 (left half) and T2 (right half). Each segment along the circle represents a B cell subset from CSF or PB. Lines connecting B cell subsets indicate B cell subsets that share 1 or more Ig-VH clusters in common. Gray lines, PB-only lg-VH clusters; blue lines, persistent CSF Ig-VH clusters; red lines, CSF Ig-VH clusters with PB contribution; yellow lines, T1-PB Ig-VH clusters that share clonal relatives with T2-CSF yet not with T1-CSF. Gray font indicates subsets or Ig isotypes from which no Ig-VH libraries could be obtained. Persistent CSF Ig-VH clusters were linked to PB Ig-VH sequences derived from Ig class-switched memory (SM) B cells ( $n=4$ patients), double-negative (DN) B cells ( $n=3$ patients at T1, $n=2$ patients at T2), and plasmablasts/plasma cells (PCs) ( $n=2$ patients at T1 and 1 patient at T2). (B) T1-PB B cell subsets that are clonally related to T2-CSF without involving T1-CSF (i.e., quantitation of yellow lines depicted in $\mathbf{A}$ ). USM, unswitched memory.

Disease-driving lymphocytes, including B cells, are believed to migrate to the CSF and CNS from peripheral stores during phases of MS disease activity $(8,11,12)$. Because of their immunological properties and functionality, SM B cells are generally believed to be highly relevant to the immune pathology of MS (35). Indeed, when persistent PB SM B cells were clonally related to second time point CSF B cells, these T2-CSF cells were nearly always SM and PC. This suggests that, in addition to CSF persistence, a population of potentially disease-relevant PB SM B cells may have a particular tendency to migrate to the CSF and mature to PCs. Also suggestive of peripheral maturation to SM B cells followed by migration and survival in the CNS, rather than local SM B cell to PC maturation in CSF/CNS, we did not find any T2-CSF B cells that were clonally related to T1-CSF naive B cells. In agreement with our previous work, we did not observe any relationships between PB-persistent PCs and CSF PCs, further supporting that migration to the CNS occurs at the SM or earlier B cell development stage followed by intrathecal maturation to PCs rather than immigration of long-lived peripheral PCs.

The efficacy of anti-CD20 B cell-depleting therapy in autoimmune disease is linked to depletion of memory B cell subsets rather than naive and transitional subsets $(36,37)$. We previously proposed that USM B cells may play a role in MS because of their appearance in MS patients' CSF during periods of active CNS inflammation (8). Interestingly, USM B cells follow the same depletion and reconstitution pattern as SM B cells under rituximab therapy (38). Here, we observed that clonally related SM and USM B cells, which remain detectable in $\mathrm{PB}$ over time, may also have clonal relatives in CSF at the later time point. This finding is consistent with the concept that, upon peripheral activation, CXCR5 ${ }^{+}$USM or SM B cells home to the brain parenchyma or leptomeninges along a CXCL13 gradient, where they participate in the formation of ectopic tertiary germinal centers $(8,9,39,40)$ capable of supporting B cell receptor affinity maturation and class-switch recombination (41). Whether USM B cell receptors undergo Ig class-switching and contribute to high-affinity surface receptor or secreted antibody-mediated immune responses has been debated $(42,43)$. Here, we found numerous Ig-VH clusters linking CSF or PB IgM-expressing USM B cells to clonally related $\mathrm{IgG}^{+} \mathrm{B}$ cells in CSF and PB (i.e., SM, PC, and DN). This finding suggests either (a) that USM B cells can enter germinal centers, where they undergo class-switch recombination or (b) that divergent maturation of naive B cells into functionally diverse IgM- and IgG-expressing memory B cell subsets occurs. Similar to SM B cells (44), USM B cells can likely function as potent antigen-presenting cells (45). Therefore, the depletion of both SM and USM B cells could be important for the efficacy of B cell-depleting MS therapies. However, whether USM B cells are involved in a true antigen-directed immune response or, conversely, whether they provide a nonspecific stimulus for intrathecal immune activation remains unclear. In either case, because of their participation in clonally related immune connectivity between PB and CSF, SM and USM B cells may be carriers of MS disease activity.

There are a number of limitations to this study. First, it is important to keep in mind that it is not technically possible to interrogate the entire immune repertoire in a living patient. As a result, persistent B cells could have gone undetected, and patients in whom we did not find clusters comprising clonally related Ig-VH may in fact harbor persistent B cells in the CSF or in other tissues. Although it is possible that deeper sequencing of existing samples could have increased our sensitivity to identify persistent CSF Ig-VH clusters, and although some samples did not generate productive sequences, we found no correlation between the average reads per cell and our ability to detect these clusters. Second, technical advances, such as incorporation of unique molecular identifiers that aid in error correction and quantification, were not a standard part of the sequencing method at the start of the study, and the initial method was maintained for consistency across this cohort. Ig-VH sequences obtained in this study overall reflected IGHV SHM accumulation as expected for the different B cell maturation states studied, with the lowest SHM in naive B cell IgM-VH (Supplemental Figure 9). The fact that some Ig-VH sequences displayed SHM counts above expected rates is likely owed to errors introduced by PCR during Ig-VH library preparation and Ion Torrent sequencing. However, PCR or sequencing errors are 
unlikely to influence determination of presence versus absence of related Ig-VH sequences but may influence the quantity of Ig-VH clusters. For this reason, we chose a distance metric (see Methods) that minimizes the risk of overestimating the number of Ig-VH clusters that could result from sequencing errors introduced by high-throughput technology. Third, although to our knowledge, this is the largest study of sorted B cell subsets from paired CSF and PB samples evaluated at 2 time points, only 10 patients were studied. Future studies will be needed to confirm these findings in larger cohorts, including patients treated with B cell-depleting therapies. Fourth, our experimental approach does not allow us to determine the directionality of B cell migration; to what extent lymphocyte migration pathways via "glymphatics" $(46,47)$ underlie our findings remains subject to future studies that will require reliable in vivo lymphocyte-tracking techniques. The question of whether clonally related B cells mature and persist in the CNS or mature and then migrate from the periphery is an important one. However, we refrained from performing mutational lineage analyses to determine B cell migration directionality because intermediate mutants that are necessary to obtain a complete picture of Ig-VH evolution may have gone undetected in our study.

In summary, we describe CSF-persistent clonally related B cell populations as part of an overall complex picture of $\mathrm{B}$ cell relatedness over time and across compartments that appears at least partially independent of IMT initiation. We identified previously unknown patterns of B cell relatedness, including PB SM and USM B cells that may contribute to SM- and PC-rich CSF B cell repertoires and naive B cells that persist over time. Our findings raise the possibility that IMTs, which effectively reduce clinical and MRI relapses, may not sufficiently target CNS-compartmentalized immune mechanisms that have been associated with gradual and relapse-independent MS worsening in relapsing MS and progressive forms of the disease (48). Effective targeting of CNS-compartmentalized inflammation may, in the future, achieve efficacy in progression slowing superior to that of current therapies (2). Longitudinal immune repertoire studies performed alongside the clinical development of such potentially novel MS drugs may well provide critical immunological and biomarker evidence indicating the successful suppression or elimination of progression-driving mechanisms.

\section{Methods}

Experimental design. We hypothesized before initiating this study that in MS patients there would be a population of B cell clones that persist in the CSF over time. Inclusion criteria for this observational study were (a) enrollment in the UCSF Expression, Proteomics, Imaging, Clinical (EPIC) study (31), which is a longitudinal MS cohort that defines MS according to the $2001 \mathrm{McDonald}$ criteria (49), and (b) CSF and PBMCs from biobanks from 2 time points (T1 and T2) taken a minimum of 9 months apart. All patients were treatment naive at T1. Patients either consented to donating excess CSF and blood during routine lumbar puncture (LP) at UCSF, or if an LP was not ordered by the treating physician, consented to undergo LP and blood draw for research purposes. Patient demographics and clinical history, including physical exam and EDSS scores, were also obtained as part of the EPIC study.

Sample collection and processing. We centrifuged 7-30 $\mathrm{ml}$ of fresh CSF at $400 \mathrm{~g} \times 15$ minutes at $4^{\circ} \mathrm{C}$ to separate a cell pellet from supernatant. PBMCs were isolated from whole blood via Ficoll gradient followed by red blood cell lysis and washing with PBS with $1 \%$ BSA. We stored 7 of 20 CSF samples and 1 of $20 \mathrm{PBMC}$ samples at $-80^{\circ} \mathrm{C}$ as unsorted cell pellets (Supplemental Table 3). The remaining CSF and PBMC samples were immediately blocked with FcR Blocking Reagent (Miltenyi Biotec) and stained with fluorescent antibodies to cell surface markers - CD19, CD27, CD38, CD138, and IgD (Supplemental Table 5); some samples were stained for additional immune cell markers for experiments outside the scope of this study (antibody panels listed in Supplemental Table 5 and Supplemental Table 6).

CSF and PB B cell subsets were sorted as previously described (8) on a Beckman MoFlo Astrios EQ FACS device: Naive B cells $\left(C D 19^{+} \operatorname{IgD}^{+} \mathrm{CD} 27^{-}\right)$, USM B cells $\left(\mathrm{CD} 19^{+} \operatorname{IgD}^{+} \mathrm{CD} 27^{+}\right)$, SM B cells $\left(\mathrm{CD} 19^{+} \operatorname{IgD}^{-} \mathrm{CD} 27^{+}\right), \mathrm{DN}$ B cells $\left(\mathrm{CD} 19^{+} \mathrm{IgD}^{-} \mathrm{CD} 27^{-}\right), \mathrm{CSF} \mathrm{CD}^{2} 7^{\text {hi }}$ plasmablasts/PCs $\left(\mathrm{CD} 19^{+} \mathrm{IgD}^{-}\right.$ $\mathrm{CD} 27^{\mathrm{hi}}$ ) (the vast majority of which are also $\left.\mathrm{CD} 38^{+}\right)$, and $\mathrm{PB}$ plasmablasts/PCs $\left(\mathrm{CD} 19^{+} \mathrm{IgD}^{-}\right.$ $\left.\mathrm{CD} 27^{+} \mathrm{CD} 38^{+}\right)$. Patient 4 had combined CD27 $7^{+/ \text {hi }} \mathrm{CSF}$-sorted B cells. CSF samples for which low B cell count was a concern were bulk sorted - flow cytometry data were attained, but all CSF lymphocytes were placed into a single sample tube rather than segregating into the above 5 subsets. B cells were sorted directly into lysis buffer suitable for later RNA extraction (Qiagen, Buffer RLT with $1 \%$ $\beta$-mercaptoethanol) and stored at $-80^{\circ} \mathrm{C}$.

Immune repertoire sequencing. RNA was extracted using the RNeasy Mini Kit for greater than 200,000 cells or the RNeasy Micro Kit for fewer than 200,000 cells (Qiagen). RNA was reverse-transcribed using 
iScript reverse transcriptase (Bio-Rad). IgG-VH and IgM-VH sequences spanning the variable region from framework 1 to the constant region 1 were amplified using the Advantage 2 PCR Kit (Clontech) and custom primers as described (8). We performed 30 PCR cycles on PB samples and 40 cycles on CSF samples; up to 10 additional cycles were performed, in 5-cycle increments, if no PCR product was visualized on a $1.5 \%$ agarose gel after the initial round of amplification. Amplicons of the expected length were gel purified and quantified by Bioanalyzer using the High Sensitivity DNA Kit (Agilent). Equimolar amounts of PCR products were pooled to generate a 16 picomolar library for Ion Torrent sequencing (Thermo Fisher Scientific) following the manufacturer's instructions, including emulsion PCR (Ion OneTouch2), enrichment for template-positive ion sphere particles, and quality control using a Qubit Fluorometer (Ion Sphere Quality Control Kit). Next-generation sequencing was performed on an IonTorrent Personal Genome Machine. If inadequate sequencing data were obtained during an initial sample's sequencing, a technical replicate was performed.

Bioinformatics. Raw sequence FASTQ files were generated with Torrent Suite software (Thermo Fisher Scientific). A custom bioinformatics pipeline incorporating MiXCR (v2.1.3) (50) was used to identify $I G H V$ and $I G H J$ germline segments and H-CDR3 for each sequence read. MiXCR first assembles reads with bases of quality scores greater than 20 and then attempts to align reads with bases of lower $(<20)$ quality to the already assembled reads. We applied a bioinformatics clustering approach as described previously (8) to compile clonally related Ig-VH sequences into "Ig-VH clusters." An Ig-VH cluster contains clonally related sequences - IgG or IgM - from at least 1 B cell. B cell receptors using identical $I G H V$ and $I G H J$ germline segments and identical or near-identical H-CDR3 regions (minimum 8 amino acid length, maximum Hamming distance of 2, ref. 51) at the amino acid level were considered members of a clonally related Ig-VH cluster. This distance metric permitted clustering of identical and clonally related $\mathrm{Ig}-\mathrm{VH}$ irrespective of sequencing errors that might have introduced a frameshift in the Ig-VH. Immune repertoire data sets can have falsely inflated diversity as a result of errors during the reverse transcription, amplification, and sequencing steps as well as incorrect barcode assignment that occurs when sequencing libraries are pooled. Thus, to conservatively catalog immune repertoire diversity in a given sample, we excluded any clusters that had fewer than 2 aligned read counts and any additional low-abundance Ig-VH clusters whose inclusion resulted in the total number of clusters exceeding the number of input cells. We excluded samples for which library generation was unsuccessful from statistical analyses.

Network diagrams were generated to visualize Ig-VH clusters using Circos (52). Amino acid alignment figures were generated using Geneious version 10.0 (created by Biomatters, https://www.geneious. com), IMGT V-QUEST (53), and NCBI IgBlast (54). Venn diagrams and other figures were based on custom R scripts that tabulated the number and type of Ig-VH clusters shared across compartments $(\mathrm{CSF} / \mathrm{PB})$ and time points.

MRI acquisition and analysis. MRI scans at T1 and T2 were acquired on the same 3T Siemens scanner following a standardized protocol that included 3D T1-weighted magnetization-prepared gradient echo images (MPRAGEs), 3D fluid-attenuated inversion recovery, and T2/proton density images of the brain and whole-spine T2-weighted and short tau inversion recovery images. In addition, brain MPRAGEs and whole-spine T1-weighted images were acquired after administration of gadobutrol. Readings were performed by a neurologist with subspecialty interest in neuroimaging ( $\mathrm{AB}, 11$ years of experience) who was blinded to the clinical and immunological data.

Data and materials availability. Ig raw sequencing data can be found at the National Center for Biotechnology Information Sequence Read Archive (BioProject accession number PRJNA517691).

Statistics. GraphPad Prism software (version 8) was used to perform statistical analyses: 2-tailed, unpaired $t$ tests were used to compare pairwise groups, and Fisher's exact test was used to compare categorical variables. One-way ANOVA was used for comparisons between groups with approximate Gaussian distributions, with correction for multiple comparisons performed using the Sidak method. Kruskal-Wallis test with Dunn's correction for multiple comparisons was used for multiple comparisons with nonparametric distributions. Spearman's correlation coefficient was used to determine correlation. $P$ value less than 0.05 was considered statistically significant.

Study approval. All studies were approved by the UCSF Institutional Review Board, and written informed consent was obtained from each participant before inclusion in the study. 


\section{Author contributions}

ALG performed immune repertoire sequencing and data analysis and wrote the manuscript. HCVB conceptualized the study, assisted in patient recruitment, provided guidance on analysis, and wrote the manuscript. MRW wrote the manuscript and provided guidance on analysis. RD and HW generated code, tables, and figures for analysis. ELE performed FACS and immune repertoire sequencing. MSW performed the OCB analysis. AB performed the analyses of MRI data. RGH oversaw acquisition of MRI data. SL performed immune repertoire sequencing and input on analysis. WH and NSP assisted in patient recruitment, consenting, and sample collection. BACC led the EPIC cohort study. SLH conceived of and led the EPIC cohort study. AR provided consultation on immune repertoire analysis and figures and reviewed the manuscript.

\section{Acknowledgments}

The authors would like to express their gratitude to the individuals who agreed to participate as patients in this study, as well as the UCSF MS EPIC study team of researchers and clinicians. Our studies were supported by grants from the National MS Society (RG-4868 to HCVB), the NIH/National Institute of Neurological Disorders and Stroke (K02NS072288 and R01NS092835 initially to HCVB, transferred to SLH; K08NS096117 to MRW), and the Valhalla Foundation, as well as by gifts from the Friends of the Multiple Sclerosis Research Group at UCSF. HCVB and MRW were also supported by an endowment from the Rachleff Family Foundation. ALG is supported by the National MS Society Kathleen C. Moore Postdoctoral Fellowship Clinician-Scientist Development Award (FAN-1507-05497).

Address correspondence to: Michael R. Wilson, 675 Nelson Rising Lane - Box 3206, San Francisco, California 94158, USA. Phone: 415.502.7429; Email: michael.wilson@ucsf.edu.

ELE's present address is: Adaptive Biotechnologies Corp., South San Francisco, California, USA. HW's present address is: Cambrian InnovaTech, Shenzhen City, Guangdong, China.

SL's present address is: Department of Neurology, University Hospital Cologne, Cologne, Germany. WH's present address is: UCSD, School of Medicine, La Jolla, California, USA.

NSP's present address is: Keck School of Medicine USC, Los Angeles, California, USA.

HCVB's present address is: F. Hoffmann-La Roche, Basel, Switzerland.

1. Molnarfi N, et al. MHC class II-dependent B cell APC function is required for induction of CNS autoimmunity independent of myelin-specific antibodies. J Exp Med. 2013;210(13):2921-2937.

2. Montalban X, et al. Ocrelizumab versus Placebo in Primary Progressive Multiple Sclerosis. N Engl J Med. 2017;376(3):209-220.

3. Hauser SL, et al. Ocrelizumab versus Interferon Beta-1a in Relapsing Multiple Sclerosis. N Engl J Med. 2017;376(3):221-234.

4. Hauser SL. The Charcot Lecture | beating MS: a story of B cells, with twists and turns. Mult Scler. 2015;21(1):8-21.

5. Dalakas MC. B cells as therapeutic targets in autoimmune neurological disorders. Nat Clin Pract Neurol. 2008;4(10):557-567.

6. 't Hart BA, et al. A new primate model for multiple sclerosis in the common marmoset. Immunol Today. 2000;21(6):290-297.

7. Hawker K, et al. Rituximab in patients with primary progressive multiple sclerosis: results of a randomized double-blind placebo-controlled multicenter trial. Ann Neurol. 2009;66(4):460-471.

8. Eggers EL, et al. Clonal relationships of CSF B cells in treatment-naive multiple sclerosis patients. JCI Insight. 2017;2(22):92724.

9. Frischer JM, et al. The relation between inflammation and neurodegeneration in multiple sclerosis brains. Brain. 2009;132(pt 5):1175-1189

10. Bevan RJ, et al. Meningeal inflammation and cortical demyelination in acute multiple sclerosis. Ann Neurol. 2018;84(6):829-842.

11. Stern JN, et al. B cells populating the multiple sclerosis brain mature in the draining cervical lymph nodes. Sci Transl Med. 2014;6(248):248ra107.

12. Palanichamy A, et al. Immunoglobulin class-switched B cells form an active immune axis between CNS and periphery in multiple sclerosis. Sci Transl Med. 2014;6(248):248ra106.

13. Bashford-Rogers RJ, et al. Eye on the B-ALL: B-cell receptor repertoires reveal persistence of numerous B-lymphoblastic leukemia subclones from diagnosis to relapse. Leukemia. 2016;30(12):2312-2321.

14. von Büdingen HC, Gulati M, Kuenzle S, Fischer K, Rupprecht TA, Goebels N. Clonally expanded plasma cells in the cerebrospinal fluid of patients with central nervous system autoimmune demyelination produce "oligoclonal bands". J Neuroimmunol. 2010;218(1-2):134-139.

15. Obermeier B, et al. Related B cell clones that populate the CSF and CNS of patients with multiple sclerosis produce CSF immunoglobulin. J Neuroimmunol. 2011;233(1-2):245-248.

16. Bankoti J, et al. In multiple sclerosis, oligoclonal bands connect to peripheral B-cell responses. Ann Neurol. 2014;75(2):266-276.

17. Yu X, et al. Intrathecally synthesized IgG in multiple sclerosis cerebrospinal fluid recognizes identical epitopes over time. 
J Neuroimmunol. 2011;240-241:129-136.

18. Walsh MJ, Tourtellotte WW. Temporal invariance and clonal uniformity of brain and cerebrospinal IgG, IgA, and IgM in multiple sclerosis. J Exp Med. 1986;163(1):41-53.

19. Glanville J, et al. Naive antibody gene-segment frequencies are heritable and unaltered by chronic lymphocyte ablation. Proc Natl Acad Sci U S A. 2011;108(50):20066-20071.

20. Colombo M, Dono M, Gazzola P, Chiorazzi N, Mancardi G, Ferrarini M. Maintenance of B lymphocyte-related clones in the cerebrospinal fluid of multiple sclerosis patients. Eur J Immunol. 2003;33(12):3433-3438.

21. Corcione A, et al. Recapitulation of B cell differentiation in the central nervous system of patients with multiple sclerosis. Proc Natl Acad Sci U S A. 2004;101(30):11064-11069.

22. Cepok S, et al. Short-lived plasma blasts are the main B cell effector subset during the course of multiple sclerosis. Brain. 2005;128(pt 7):1667-1676.

23. Taylor JJ, Pape KA, Jenkins MK. A germinal center-independent pathway generates unswitched memory B cells early in the primary response. J Exp Med. 2012;209(3):597-606.

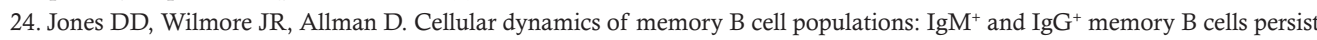
indefinitely as quiescent cells. J Immunol. 2015;195(10):4753-4759.

25. Magliozzi R, et al. Meningeal B-cell follicles in secondary progressive multiple sclerosis associate with early onset of disease and severe cortical pathology. Brain. 2007;130(pt 4):1089-1104.

26. Confavreux C, Chapuis-Cellier C, Arnaud P, Robert O, Aimard G, Devic M. Oligoclonal "fingerprint" of CSF IgG in multiple sclerosis patients is not modified following intrathecal administration of natural $\beta$-interferon. J Neurol Neurosurg Psychiatry. 1986;49(11):1308-1312.

27. Li R, Bar-Or A. The multiple roles of B cells in multiple sclerosis and their implications in multiple sclerosis therapies [published online ahead of print April 16, 2018]. Cold Spring Harb Perspect Med. https://doi.org/10.1101/cshperspect.a029108.

28. Pitzalis C, Jones GW, Bombardieri M, Jones SA. Ectopic lymphoid-like structures in infection, cancer and autoimmunity. Nat Rev Immunol. 2014;14(7):447-462.

29. Lovato L, et al. Related B cell clones populate the meninges and parenchyma of patients with multiple sclerosis. Brain. 2011;134(pt 2):534-541.

30. von Büdingen HC, et al. Onset of secondary progressive MS after long-term rituximab therapy — a case report. Ann Clin Transl Neurol. 2017;4(1):46-52.

31. University of California, San Francisco MS-EPIC Team, et al. Long-term evolution of multiple sclerosis disability in the treatment era. Ann Neurol. 2016;80(4):499-510.

32. Komori M, et al. Insufficient disease inhibition by intrathecal rituximab in progressive multiple sclerosis. Ann Clin Transl Neurol. 2016;3(3):166-179.

33. Studer V, Rossi S, Motta C, Buttari F, Centonze D. Peripheral B cell depletion and central proinflammatory cytokine reduction following repeated intrathecal administration of rituximab in progressive Multiple Sclerosis. J Neuroimmunol. 2014;276(1-2):229-231.

34. Piccio L, et al. Changes in B- and T-lymphocyte and chemokine levels with rituximab treatment in multiple sclerosis. Arch Neurol. 2010;67(6):707-714.

35. Baker D, Marta M, Pryce G, Giovannoni G, Schmierer K. Memory B Cells are Major Targets for Effective Immunotherapy in Relapsing Multiple Sclerosis. EBioMedicine. 2017;16:41-50.

36. Roll P, Palanichamy A, Kneitz C, Dorner T, Tony HP. Regeneration of B cell subsets after transient B cell depletion using anti-CD20 antibodies in rheumatoid arthritis. Arthritis Rheum. 2006;54(8):2377-2386.

37. Greenfield AL, Hauser SL. B-cell Therapy for Multiple Sclerosis: Entering an era. Ann Neurol. 2018;83(1):13-26.

38. Colucci M, et al. B Cell Reconstitution after Rituximab Treatment in Idiopathic Nephrotic Syndrome. J Am Soc Nephrol. 2016;27(6):1811-1822.

39. Magliozzi R, Columba-Cabezas S, Serafini B, Aloisi F. Intracerebral expression of CXCL13 and BAFF is accompanied by formation of lymphoid follicle-like structures in the meninges of mice with relapsing experimental autoimmune encephalomyelitis. J Neuroimmunol. 2004;148(1-2):11-23.

40. Serafini B, Rosicarelli B, Magliozzi R, Stigliano E, Aloisi F. Detection of ectopic B-cell follicles with germinal centers in the meninges of patients with secondary progressive multiple sclerosis. Brain Pathol. 2004;14(2):164-174.

41. Lehmann-Horn K, Wang SZ, Sagan SA, Zamvil SS, von Büdingen HC. B cell repertoire expansion occurs in meningeal ectopic lymphoid tissue. JCI Insight. 2016;1(20):e87234.

42. Horns F, et al. Lineage tracing of human B cells reveals the in vivo landscape of human antibody class switching. Elife. 2016;5:e16578.

43. Seifert M, et al. Functional capacities of human IgM memory B cells in early inflammatory responses and secondary germinal center reactions. Proc Natl Acad Sci U S A. 2015;112(6):E546-E555.

44. Bar-Or A, et al. Immunological memory: contribution of memory B cells expressing costimulatory molecules in the resting state. J Immunol. 2001;167(10):5669-5677.

45. Abramowski P, Otto B, Martin R. The orally available, synthetic ether lipid edelfosine inhibits T cell proliferation and induces a type I interferon response. PLoS One. 2014;9(3):e91970.

46. Abbott NJ, Pizzo ME, Preston JE, Janigro D, Thorne RG. The role of brain barriers in fluid movement in the CNS: is there a 'glymphatic' system? Acta Neuropathol. 2018;135(3):387-407.

47. Engelhardt B, Carare RO, Bechmann I, Flügel A, Laman JD, Weller RO. Vascular, glial, and lymphatic immune gateways of the central nervous system. Acta Neuropathol. 2016;132(3):317-338.

48. Kappos L, et al. Greater sensitivity to multiple sclerosis disability worsening and progression events using a roving versus a fixed reference value in a prospective cohort study. Mult Scler. 2018;24(7):963-973.

49. McDonald WI, et al. Recommended diagnostic criteria for multiple sclerosis: guidelines from the International Panel on the diagnosis of multiple sclerosis. Ann Neurol. 2001;50(1):121-127.

50. Bolotin DA, et al. MiXCR: software for comprehensive adaptive immunity profiling. Nat Methods. 2015;12(5):380-381. 
51. Hamming RW. Error Detecting and Error Correcting Codes. Bell Syst Tech J. 1950;29(2):147-160.

52. Krzywinski M, et al. Circos: an information aesthetic for comparative genomics. Genome Res. 2009;19(9):1639-1645.

53. Giudicelli V, Brochet X, Lefranc MP. IMGT/V-QUEST: IMGT standardized analysis of the immunoglobulin (IG) and T cell receptor (TR) nucleotide sequences. Cold Spring Harb Protoc. 2011;2011(6):695-715.

54. Ye J, Ma N, Madden TL, Ostell JM. IgBLAST: an immunoglobulin variable domain sequence analysis tool. Nucleic Acids Res. 2013;41(Web Server issue):W34-W40 